\title{
Anodic Oxidation of Titanium in Sulphuric Acid and Phosphoric Acid Electrolytes
}

\author{
Uttiya $S^{1}$, Contarino $\mathrm{D}^{1}$, Prandi $\mathrm{S}^{2}$, Carnasciali $\mathrm{MM}^{3}$, Gemme $\mathrm{G}^{4}$, Mattera $\mathrm{L}^{1}$, Rolandi $\mathrm{R}^{1}$, Canepa $\mathrm{M}^{1}$ and \\ Cavalleri $\mathrm{O}^{*}$
}

${ }^{1}$ Department of Physics, University of Genova, Italy

${ }^{2}$ ARPAL, Department of Genova, Italy

${ }^{3}$ Department of Chemistry and Industrial Chemistry, University of Genova, Italy

${ }^{4}$ INFN, Genoa Unit, Italy

*Corresponding author: Cavalleri O, Department of Physics, University of Genova, Italy, E-mail: cavalleri@ fisica.unige.it

Citation: Uttiya S, Contarino D, Prandi S, Carnasciali MM, Gemme G, et al. (2014) Anodic Oxidation of Titanium in Sulphuric Acid and Phosphoric Acid Electrolytes. J Mater Sci Nanotechnol 1(1): S106. doi: 10.15744/2348-9812.1.S106

Received Date: June 07, 2014 Accepted Date: June 11, 2014 Published Date: July 18, 2014

\begin{abstract}
Anodisation of pure titanium has been carried out in sulphuric and in phosphoric acid solutions at potentials ranging from 50 to $150 \mathrm{~V}$. The SEM and AFM morphological analysis indicates that, within this potential range, oxidation in sulphuric acid solution produces better developed mesoporous oxide layers. XPS analysis of the Ti2p core level region and Raman spectroscopy measurements show that oxide layers grown in sulphuric acid are characterised by a better defined oxidation state of titanium and by a higher content in anatase crystalline phase. XPS measurements indicate that oxidation in phosphoric acid solution produces oxide layers with two components of stoichiometric oxide and with the presence of phosphate inclusions which can be interesting in view of biomedical applications.
\end{abstract}

\section{Introduction}

Titanium and its alloys are largely employed for the development of orthopaedic and dental implants because of their good mechanical strength and low density. Thanks to its thin native oxide layer which prevents ion release, titanium offers good resistance to corrosion in body fluids and good biocompatibility. These properties can be further improved by surface treatments able to grow thicker oxide layers with controlled morphological and physico-chemical properties. Several methods have been used to this purpose, from thermal treatment [1-3], to acid solution immersion [4], peroxide immersion [5], anodic-plasma chemical treatment [6], plasma immersion ion implantation [7] and anodisation [8,9].

In recent years anodisation has been widely used to grow oxide layers on titanium [10]. Different morphologies, crystalline phases and selective enrichment with chemical species have been obtained by tuning anodising conditions and post anodisation treatments [11]. In particular anodisation in fluoride ion containing electrolytes results in the formation of nanotubular $\mathrm{TiO}_{2}$ layers [12,13] while nanoporous oxide layers can be grown in acidic electrolytes in the absence of fluoride compounds [14,15]. Depending on the anodising protocols, the formation of amorphous and/or crystalline oxide layers has been reported as well as the annealing induced inter-phase conversion [16,17]. In view of biomedical applications, to enhance the osseintegration properties of the oxide layer, different strategies have been explored, including coating with hydroxyapatite [18] or anodisation in Ca or P containing electrolytes to obtain enriched bioactive surfaces [6].

In the present study we investigated the anodisation of titanium in sulphuric and phosphoric acid solutions at different anodising potentials with the aim of correlating the oxide morphological and structural properties with the anodisation conditions. This work is part of an ongoing project focussed on the growth and functionalization of titanium oxide layers aimed to develop $\mathrm{TiO}_{2}$ surfaces with improved antibacterial properties.

\section{Experimental}

Titanium foil (purity 99.7\%), $1 \mathrm{~mm}$ in thickness, was purchased from Mateck GmbH (Germany) and cut in $10 \mathrm{~mm}$ x $10 \mathrm{~mm}$ specimens. Prior to anodisation samples were mechanically polished with SiC abrasive paper (from P800 to P4000 grade). After polishing samples were sonicated in acetone, ethanol and Milli-Q water, successively, and dried in a nitrogen stream. 
Polished samples were anodised in a two-electrode cell, using a Pt grid as a counter-electrode. Anodisation was performed in $1 \mathrm{M}$ $\mathrm{H}_{2} \mathrm{SO}_{4}$ and $1.5 \mathrm{M} \mathrm{H}_{3} \mathrm{PO}_{4}$ for $1 \mathrm{~min}$ at voltages of $50 \mathrm{~V}, 70 \mathrm{~V}, 100 \mathrm{~V}, 120 \mathrm{~V}$ and $150 \mathrm{~V}$. The electrolytes were prepared from $98 \% \mathrm{H}_{2} \mathrm{SO}_{4}$ (p.a. Fluka) and $85 \% \mathrm{H}_{3} \mathrm{PO}_{4}$ (p.a. Merck), respectively. Oxidized samples were thoroughly rinsed with Milli-Q water and dried. In order to investigate possible structural changes of the anodic oxides with annealing, some anodised samples were annealed in air at $600{ }^{\circ} \mathrm{C}$ for 1 hour.

The morphology of oxidised samples was investigated by scanning electron microscopy (SEM) and tapping mode atomic force microscopy (AFM). SEM measurements were performed using a LEO1450VP SEM with tungsten filament. The images were obtained with the signal of secondary electrons. The analysis was carried out in high vacuum conditions, without any metallization of the sample. SEM images were analysed using ImageJ software. The equivalent pore diameter was calculated as the average between the pore widths measured along the maximum and minimum pore axes. In the histograms, the number of bins was set to the integer closest to $\sqrt{ } \mathrm{N}$. Tapping mode AFM measurements were performed using a Dimension 3100/Nanoscope III system (Digital Instruments-Bruker) and Si cantilevers (OMCLAC160TS, Olympus).

The chemical analysis of the sample surfaces has been carried out by XPS measurements using a 5600 MultiTechnique apparatus operated as reported in a previous study [19]. An X-ray Al- monochromatised source $(h v=1486.6 \mathrm{eV})$ was used. The binding energy scale was referenced by setting the $\mathrm{C} 1 \mathrm{~s}$ of adventitious carbon at $284.8 \mathrm{eV}$.

Micro Raman analyses were carried out with a Renishaw System 2000 spectrometer equipped with a Peltier cooled CCD detector and coupled with a Leica optical microscope. The red line at $632.8 \mathrm{~nm}$ of a He-Ne laser was used as the excitation source, with laser power kept as low as possible to avoid thermal heating of the sample.

\section{Results and Discussions}

SEM and AFM analysis was carried out to investigate the surface morphology of anodised samples as a function of the applied voltage for oxide layers grown in sulphuric and in phosphoric acid electrolytes.

The overall analysis of the surface morphology of samples oxidized in $\mathrm{H}_{2} \mathrm{SO}_{4}$ indicates the formation of a mesoporous oxide layer. Two representative SEM images of samples oxidized at $100 \mathrm{~V}$ and $150 \mathrm{~V}$ are shown in Figure 1a and 1c. At $100 \mathrm{~V}$, isolated pores are formed. Most of them are roundly shaped and, for some pores, a sort of pore wall can be distinguished around the cavity (better observed in AFM images, see Figure 2). Figure 1e shows the histogram of the equivalent pore diameters. A Gaussian function was fitted to the histogram by using central value and $\sigma$ as fitting parameters. The distribution central value and $\sigma$ turn out to be $(71 \pm 3) \mathrm{nm}$ and $(46 \pm 7) \mathrm{nm}$, respectively. Increasing the anodisation potential up to 150V results in the formation of larger pores (Figure 1c). Some of them have an elongated shape and a multi-pore structure and are likely the result of a coalescence process; as a consequence, the density of pores on the surface decreases. The histogram of the equivalent pore diameters is shown in Figure 1f. Again a Gaussian function was fitted to the histogram. The distribution central value and $\sigma$ result to be (254 \pm 9$) \mathrm{nm}$ and $(79 \pm 16) \mathrm{nm}$, respectively. The changes in pore size and morphology observed by SEM are confirmed by AFM analysis which allows to investigate in more detail the surface morphology and to quantify the vertical excursions of the surface topography. Figure 2 a, $c$, e shows a series of AFM images acquired on samples oxidized in $\mathrm{H}_{2} \mathrm{SO}_{4}$ at increasing voltages: $100 \mathrm{~V}, 120 \mathrm{~V}$ and $150 \mathrm{~V}$. Anodisation at $100 \mathrm{~V}$ produces oxide layers characterized by individual pores with clearly distinguishable wall boundaries. Very similar pore morphologies are observed on samples anodised at 70V (data not shown). The apparent depth of the pores measured with respect to the wall is of the order of 150-200 nm. It must be noted that the hole depth values measured on AFM images represent a lower limit of the real hole depth because of the convolution effect due to the finite size of the AFM tip. When the anodisation potential is increased, single pore boundaries are no more easily distinguishable and on samples oxidised at $150 \mathrm{~V}$ pores seem to be formed on an almost continuous ground layer. The maximum apparent hole depths measured on samples prepared at $150 \mathrm{~V}$ are in the range 500-600 nm, consistently with the fact that a minor depth underestimation is expected for larger pores.

The oxide layers formed upon anodisation in $\mathrm{H}_{3} \mathrm{PO}_{4}$ are less homogeneous compared to those grown in $\mathrm{H}_{2} \mathrm{SO}_{4}$ solutions. The combined SEM/AFM analysis shows that regions with well-defined pores alternate with regions with not completely formed pores. Two representative SEM images of samples oxidized in $\mathrm{H}_{3} \mathrm{PO}_{4}$ at $100 \mathrm{~V}$ and $150 \mathrm{~V}$ are shown in Figure $1 \mathrm{~b}$ and $1 \mathrm{~d}$. The density of well- defined pores is lower compared to samples oxidised in sulphuric acid, but in a similar way to what is observed upon oxidation in sulphuric acid, pores formed at $100 \mathrm{~V}$ are surrounded by a sort of wall boundary which can not be distinguished around pores formed at 150V. AFM images (Figure $2 \mathrm{~b}, \mathrm{~d}, \mathrm{f}$ ) provide a direct 3D evaluation of the topography of the sample morphology. Samples oxidised at potentials lower than $100 \mathrm{~V}$ (data not shown) are characterised by the presence of oxide clusters and only a few pits can be observed on the surface. This is in agreement with a previous study on titanium anodisation in phosphoric acid at low voltages $(3-30 \mathrm{~V})$ which reported the formation of globular structures [20]. Increasing the anodisation potential up to 100-120V produces oxide layers characterised by pores, quite irregular in shape, coexisting with clusters. A more uniform surface morphology is observed on samples oxidised at $150 \mathrm{~V}$ even though some clusters are still present on the sample and coexist with porous areas. The comparison of Figure $2 \mathrm{e}$ and $\mathrm{f}$ shows that pores formed in phosphoric acid are smaller than those formed in sulphuric acid. 
(a)

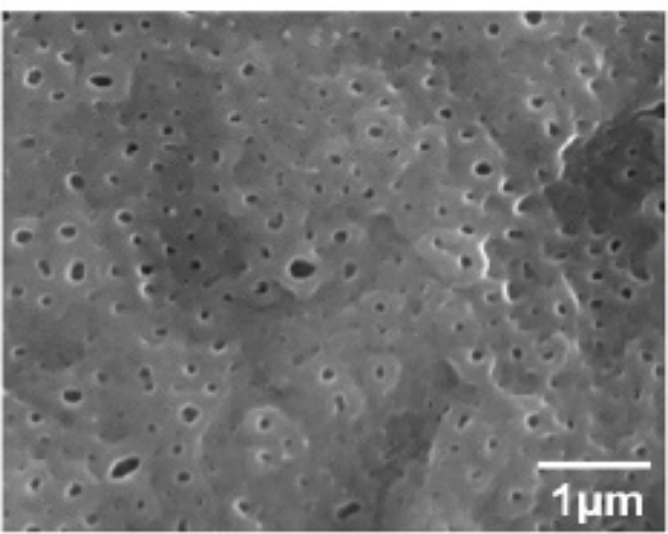

(c)

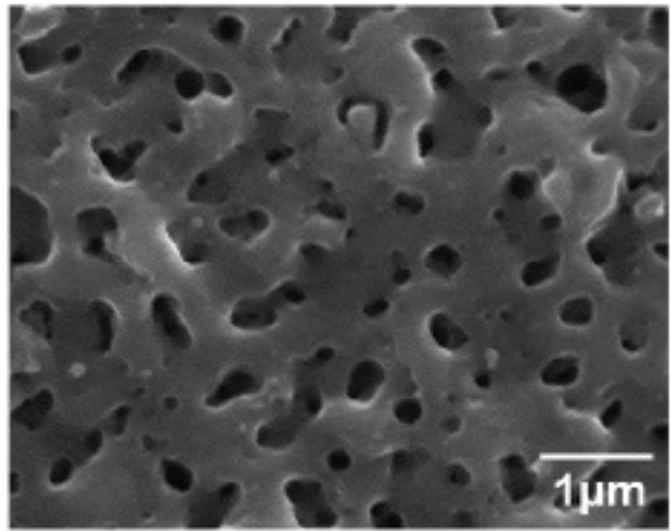

(e)

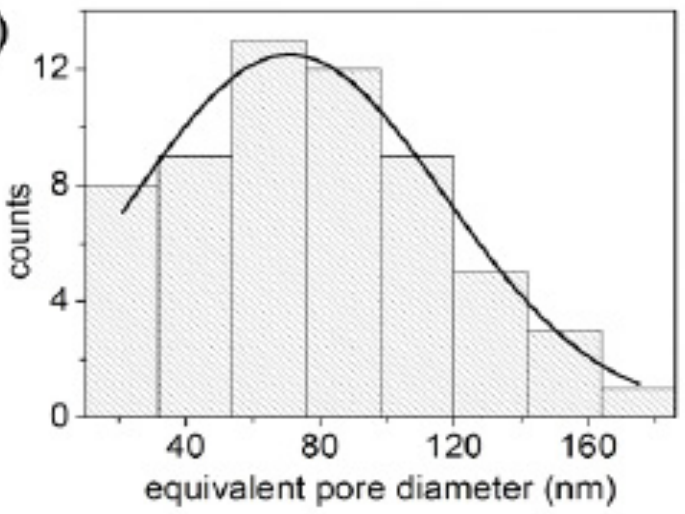

(b)

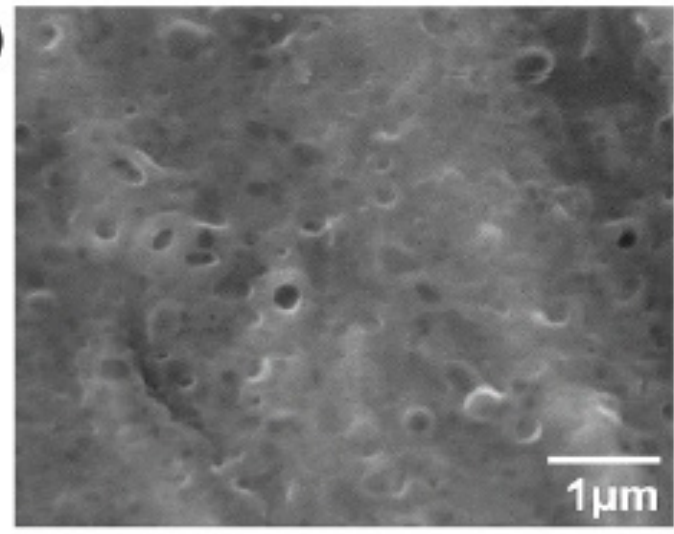

(d)

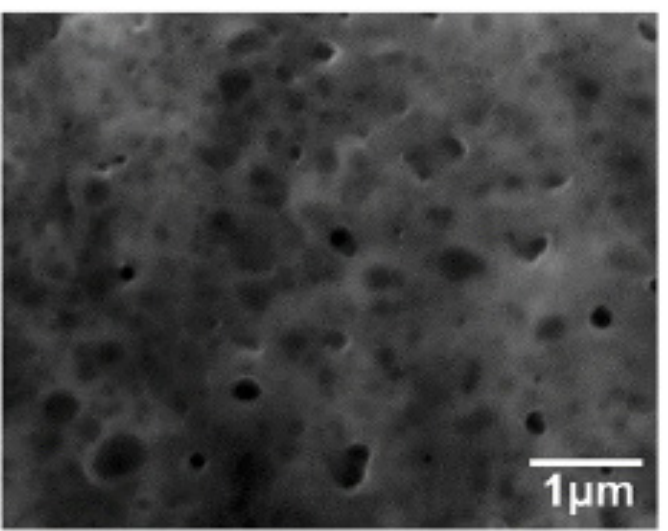

(f)

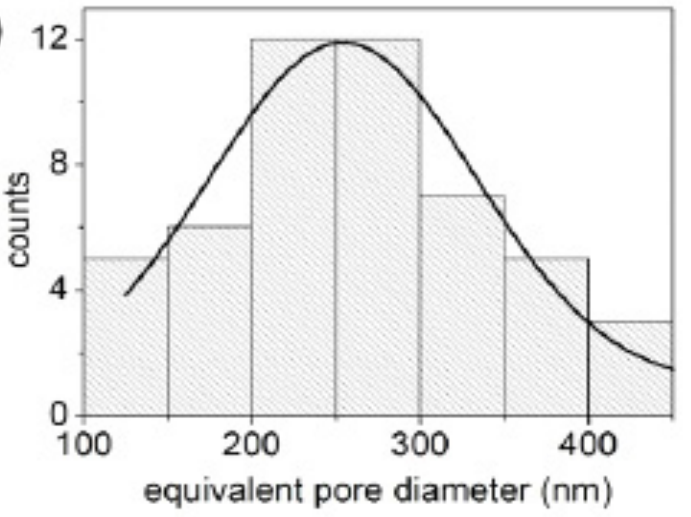

Figure 1: SEM images of titanium samples after anodisation in (a) $\mathrm{H}_{2} \mathrm{SO}_{4}$ at $100 \mathrm{~V}$, (b) $\mathrm{H}_{3} \mathrm{PO}_{4}$ at $100 \mathrm{~V},(\mathrm{c}) \mathrm{H}_{2} \mathrm{SO}_{4}$ at $150 \mathrm{~V}$, (d) $\mathrm{H}_{3} \mathrm{PO}_{4}$ at $150 \mathrm{~V}$, (e) histogram of the equivalent pore diameters of samples anodised in $\mathrm{H}_{2} \mathrm{SO}_{4}$ at $100 \mathrm{~V}$, (f) ) histogram of the equivalent pore diameters of samples anodised in $\mathrm{H}_{2} \mathrm{SO}_{4}$ at $150 \mathrm{~V}$.

The comparative morphological analysis of oxide layers formed in sulphuric and phosphoric acid as a function of the anodisation potential indicates that lower potentials are needed to achieve the formation of well-defined mesoporous layers when anodisation is carried out in sulphuric acid. This finding is in agreement with a previous study on the anodic oxidation of titanium in phosphoric acid that reports the formation of porous layer for potentials from $150 \mathrm{~V}$ to $250 \mathrm{~V}$ [15] while well- defined microporous layers have been observed upon anodisation at $150 \mathrm{~V}$ in sulphuric acid [14].

The observation that higher voltages are necessary to develop porous layers in phosphoric acid with respect to sulphuric acid can be related to the fact that the onset of pore formation occurs when the applied potential is high enough to cause a dielectric breakdown of the oxide layer. The dielectric breakdown voltage depends on several parameters, including solution $\mathrm{pH}$, temperature and conductivity. At present we do not have systematic data on the dependence of the onset of pore formation on such parameters. However the observation, for $\mathrm{M}$ solutions, of higher breakdown potentials in phosphoric acid solution than in sulphuric acid solutions [21] could account for the differences in the pore forming potentials observed in the present study. 

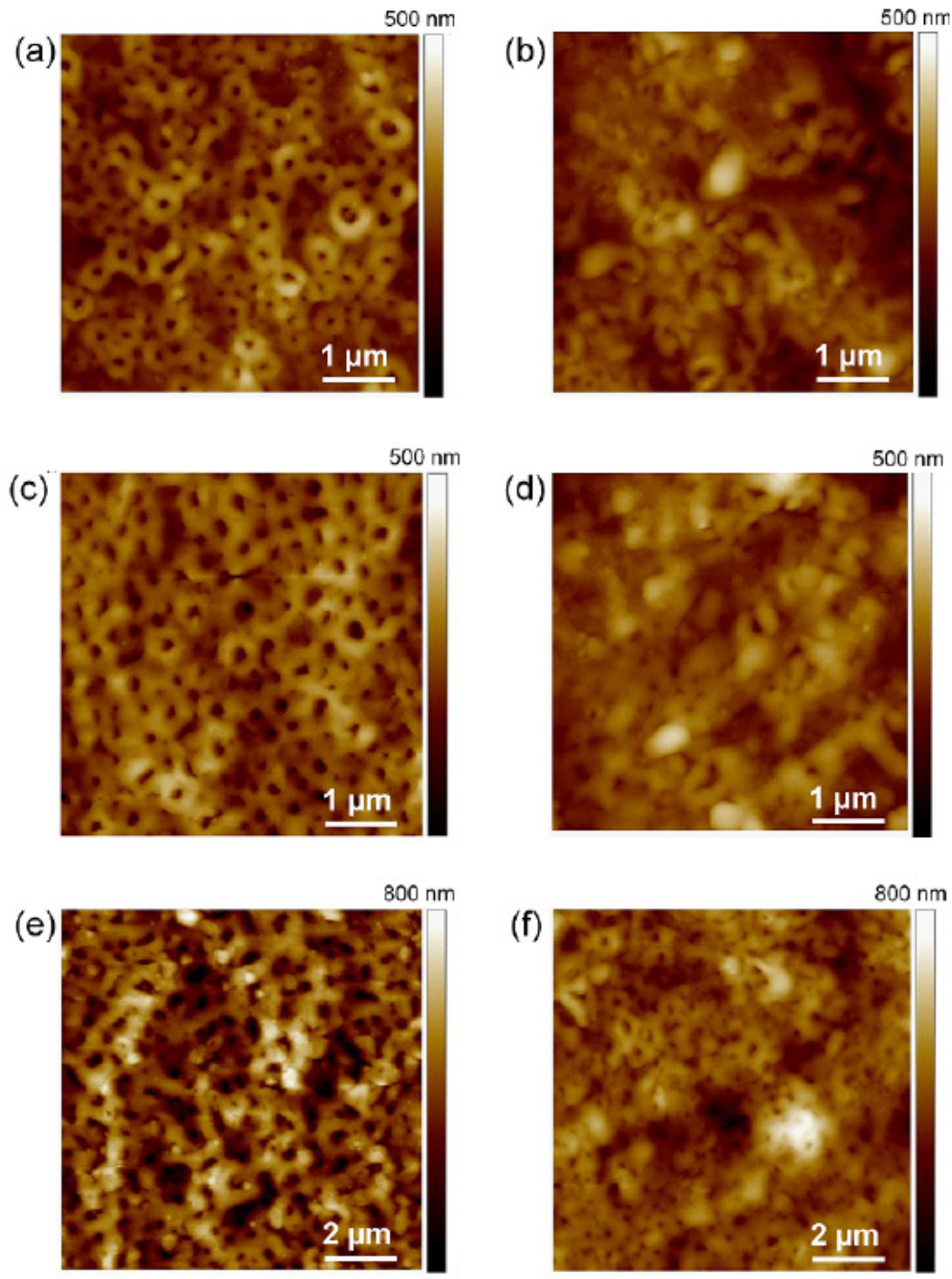

Figure 2: AFM images of titanium samples after anodisation in (a) $\mathrm{H}_{2} \mathrm{SO}_{4}$ at $100 \mathrm{~V}$, (b) $\mathrm{H}_{3} \mathrm{PO}_{4}$ at $100 \mathrm{~V}$, (c) $\mathrm{H}_{2} \mathrm{SO}_{4}$ at $120 \mathrm{~V}$, (d) $\mathrm{H}_{3} \mathrm{PO}_{4}$ at $120 \mathrm{~V}$, (e) $\mathrm{H}_{2} \mathrm{SO}_{4}$ at $150 \mathrm{~V}$, (f) $\mathrm{H}_{3} \mathrm{PO}_{4}$ at $150 \mathrm{~V}$. 
Figure 3a shows the XPS survey spectra of titanium after anodisation in sulphuric (blue curve) and phosphoric (red curve) acid solutions. Peaks corresponding to Ti, $\mathrm{O}$ and $\mathrm{C}$ are present in both spectra. P peaks can be observed in the spectrum of Ti oxidized in phosphoric acid electrolytes while no $\mathrm{S}$ signal can be detected from the analysis of samples oxidized in sulphuric acid electrolytes. The lower surface concentration of electrolyte inclusions detected on samples anodised in sulphuric acid with respect to samples anodised in phosphoric acid is consistent with a chemical depth profiling study performed by glow discharge optical emission spectroscopy (GDOES) on titanium oxidised in sulphuric and phosphoric acid [22]. The GDOES profiles of samples oxidised at $20 \mathrm{~V}$ and $80 \mathrm{~V}$ indicate that the $\mathrm{S}$ concentration is lower than the $\mathrm{P}$ concentration. Moreover, the $\mathrm{P}$ concentration profile has a maximum at the oxide surface while the maximum of the $\mathrm{S}$ concentration is localised some tens of nm below the surface. It is therefore reasonable that no S signal can be detected by a surface technique like XPS. Minor Ca and N peaks are occasionally present in the spectra (as can be observed in Figure 3a, red curve). Ca impurities are likely due to the polishing process.
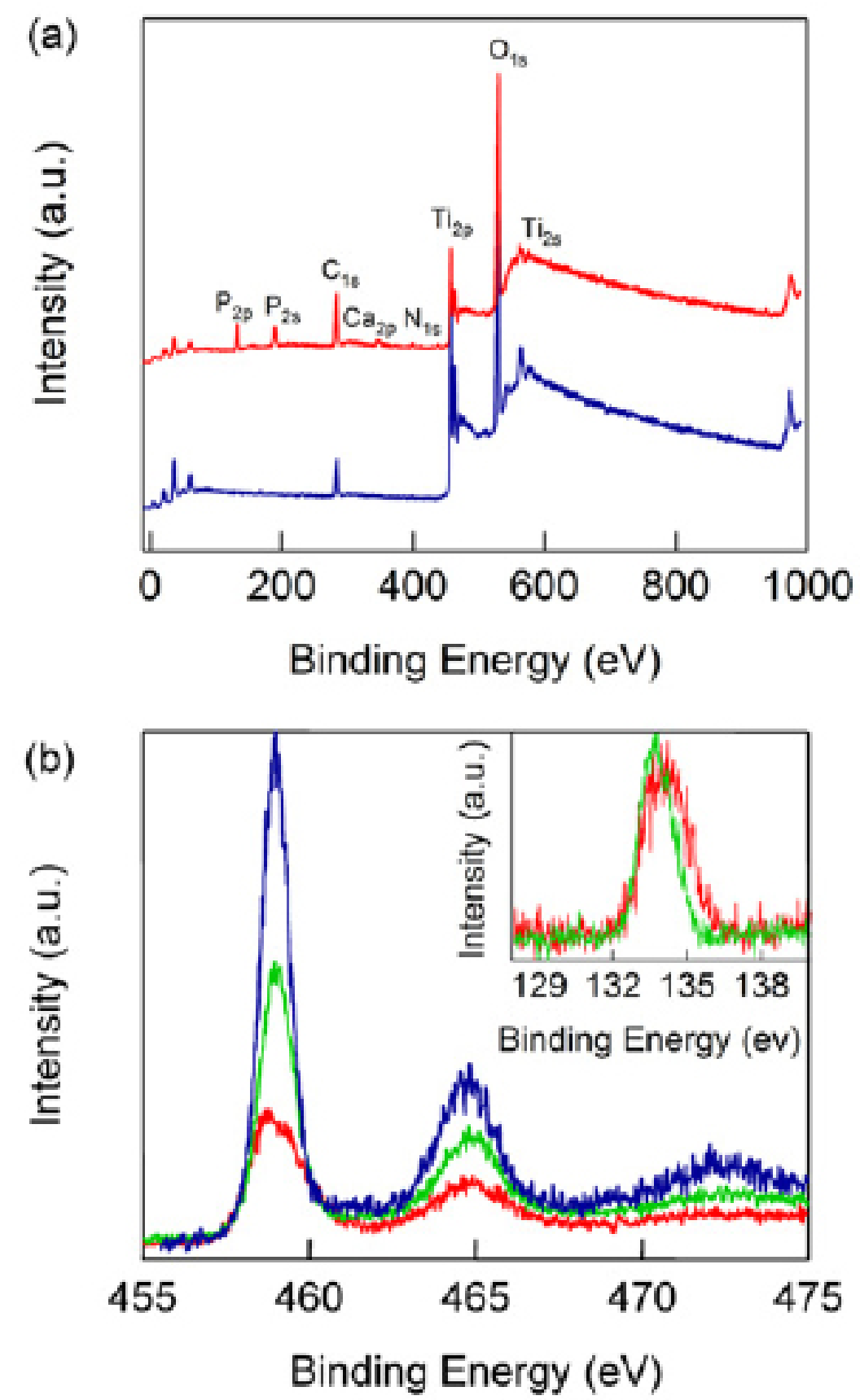

Figure 3: (a) XPS survey spectra of titanium after anodisation in $\mathrm{H}_{2} \mathrm{SO}_{4}$ (blue curve) and $\mathrm{H}_{3} \mathrm{PO}_{4}$ (red curve) at $120 \mathrm{~V}$. (b) Ti2 $\mathrm{p}$ core level region of a titanium sample after anodisation in: $\mathrm{H}_{2} \mathrm{SO}_{4}$ at $120 \mathrm{~V}$ (blue curve), $\mathrm{H}_{3} \mathrm{PO}_{4}$ at $120 \mathrm{~V}$ (red curve), $\mathrm{H}_{3} \mathrm{PO}_{4}$ at $120 \mathrm{~V}$ after 1 hour annealing at $600{ }^{\circ} \mathrm{C}$ (green curve). Inset: $\mathrm{P} 2 \mathrm{p}$ core level region of a sample after anodisation in: $\mathrm{H}_{3} \mathrm{PO}_{4}$ at $120 \mathrm{~V}$ (red curve), $\mathrm{H}_{3} \mathrm{PO}_{4}$ at $120 \mathrm{~V}$ after 1 hour annealing at $600{ }^{\circ} \mathrm{C}$ (green curve). 
The Ti2p core level region spectra of Ti after anodisation in sulphuric (blue curve) and phosphoric (red curve) acid solutions are reported in Figure $3 b$. To address the effect of thermal annealing on the chemical species present on the sample surface, the Ti2p spectrum acquired on a sample anodised in phosphoric acid and subsequently annealed is reported for comparison (green curve). The Ti2 $\mathrm{p}$ spectrum of Ti anodised in sulphuric acid is characterised by a well-defined $2 \mathrm{p}$ doublet with the $2 \mathrm{p}_{3 / 2}$ component at $(459.0 \pm 0.2) \mathrm{eV}$. From the comparison with previous studies on titanium dioxide [23-26], the Ti2p signal can be attributed to $\mathrm{Ti}^{4+}$, indicating the formation of a stoichiometric oxide. The Ti2p spectrum of titanium samples oxidised in phosphoric acid is characterised by a broader peak, which is likely due to the presence of two Ti2p components. The binding energies of the two components are within the energy range of $\mathrm{Ti}^{4+}$ and suggest the presence of two different forms of stoichiometric oxides, likely related to the nanostructuring. Annealing results in a narrowing of the $\operatorname{Ti} 2 \mathrm{p}_{3 / 2}$ peak suggesting some reordering of the oxide structure. As can be observed in the survey spectrum (Figure 3a, red curve), anodisation in phosphoric acid produces oxide layers with surface $\mathrm{P}$ inclusions. The P2p core level region (Figure 3b, inset) shows a peak at about $134 \mathrm{eV}$ that can be assigned to phosphate species [27]. P incorporation in Ti anodised in phosphoric acid has been previously reported in XPS studies of anodisation at low voltages [20] and in galvanostatic conditions [28]. The formation of titanium phosphate compounds has been reported in a X-ray diffraction study on anodised titanium [21]. As observed for the Ti2p spectrum, annealing produces some peak narrowing also in the P2p signal, suggesting some structural reordering of the oxide layer.

To investigate the crystalline structure of the oxide layers we performed Raman spectroscopy measurements. The Raman spectra of Ti anodised in sulphuric and phosphoric acid at $120 \mathrm{~V}$, prior and after 1 hour annealing at $600{ }^{\circ} \mathrm{C}$ are reported in Figure 4 . The

(a)

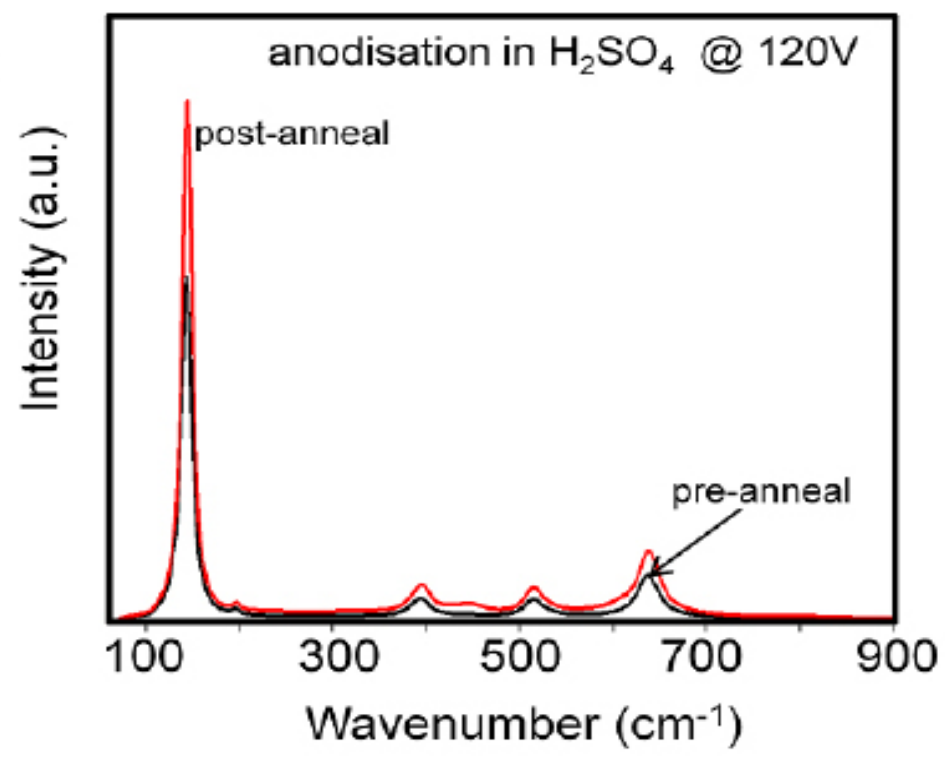

(b)

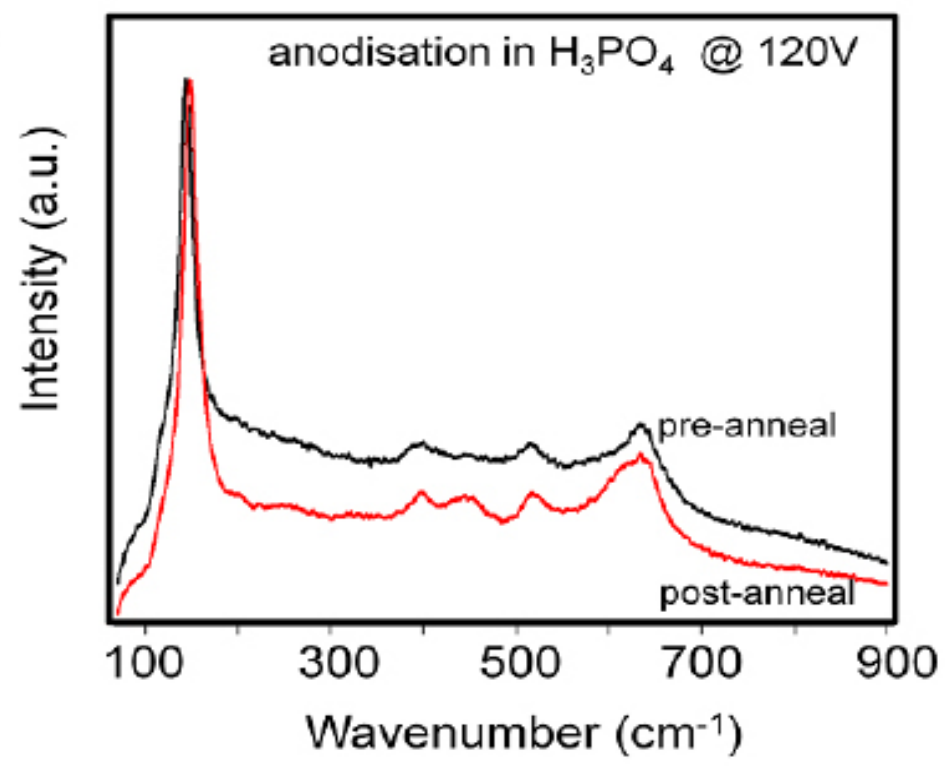

Figure 4: Raman spectra of titanium samples after anodisation in: (a) $\mathrm{H}_{2} \mathrm{SO}_{4}$ at $120 \mathrm{~V}$, (b) $\mathrm{H}_{3} \mathrm{PO}_{4}$ at $120 \mathrm{~V}$, before and after 1 hour annealing at $600{ }^{\circ} \mathrm{C}$. 
spectrum of titanium oxidised in sulphuric acid (Figure 4a) presents the characteristic peaks at 144, 197, 396,515 and $638 \mathrm{~cm}^{-1}$ of the anatase phase $[29,30]$ in agreement with the results reported by Jaeggi et al. [22]. The peak intensities increase after annealing indicating a growth of the anatase phase. Titanium anodised in phosphoric acid (Figure $4 \mathrm{~b}$ ) is characterised by a less defined crystalline structure with some broad peaks assignable to the anatase phase, but the overall spectrum is indicative of the formation of an oxide layer with a significant amorphous phase. Also in this case, annealing contributes to improve the sample crystalline order with a slight increase in peak intensity and with the appearance of two spectral features, a shoulder of the $640 \mathrm{~cm}^{-1}$ peak and a peak around $450 \mathrm{~cm}^{-1}$, that could be related to a small content of rutile phase [29]. Indeed transition from anatase to rutile has been reported for both porous and tubular $\mathrm{TiO}_{2}$ layers, even though different anatase to rutile transition temperatures, from about $450^{\circ} \mathrm{C}$ to about $700{ }^{\circ} \mathrm{C}$, have been reported depending on the specific anodising conditions used for the $\mathrm{TiO}_{2}$ preparation $[11,16,17]$.

\section{Conclusions}

From the comparative morphological and spectroscopic investigation of titanium samples oxidised in sulphuric and phosphoric acid it turns out that oxide layers grown in sulphuric acid are more uniform with respect to those obtained using phosphoric acid: i) they have a more defined mesoporous morphology, ii) the narrower Ti2p XPS spectrum indicates the formation of an oxide layer with a better defined Ti oxidation state, iii) Raman measurements indicate the formation of oxide layers with a prevalent anatase crystalline phase.

As concerns oxidation in phosphoric acid, the XPS Ti2p spectrum shows the presence of two components both related to stoichiometric oxide. These two components are likely related to a different sample nanostructuring. A narrowing of the Ti2 $p$ spectrum is observed upon annealing. The presence of the two components and their evolution with annealing as well as their relationship with the anodising potential is an interesting aspect which deserves further investigations. Another interesting aspect of samples prepared in phosphoric acid is related to the presence of phosphate inclusions which can be useful in view of biomedical application [31]. Under this perspective, oxidation in phosphoric acid solution carried out at higher anodising potentials represents a viable strategy to prepare well-developed mesoporous structures with a higher phosphate content. Having in mind the developments of biomaterials for implants, a further issue to be addressed will be the design of proper oxide surface treatments able to promote resistance to bacterial adhesion by either using antifouling-antibacterial coatings [32] or loading the pores with biocidic nanoparticles [33].

\section{Acknowledgements}

The authors gratefully acknowledge Dr. Francesco Bisio (CNR-SPIN, Genoa) for assistance with the XPS measurements, Dr. Amanda Penco (DIFI, Genoa) for assistance with the AFM measurements, Dr. Marcella Pani (DCCI, Genoa) for help with annealing experiments, Dr. Marco Salerno (IIT, Genoa) and Dr. Antonio Barbucci (DICCA, Genoa) for fruitful discussion on anodic oxidation. S.U. acknowledges financial support from "Fondo Sociale Europeo Regione Liguria” (project n. DPU12UNIGE82/3600).

\section{References}

1. Kumar S, Sankara Narayanan TSN, Ganesh Sundara Raman S, Seshadri SK (2010) Surface modification of CP-Ti to improve the fretting-corrosion resistance: Thermal oxidation vs. anodizing. Materials Science and Engineering C 30: 921-7.

2. Ishizawa H, Ogino M (1995) Characterization of thin hydroxyapatite layers formed on anodic titanium oxide films containing Ca and P by hydrothermal treatment. J Biomed Mater Res 29: 1071-9.

3. Fini M, Cigada A, Rondelli G, Chiesa R, Giardino R, et al. (1999) In vitro and in vivo behaviour of Ca- and P-enriched anodized titanium. Biomaterials 20: 1587-94.

4. Park JW, Kim YJ, Jang JH, Kwon TG, Bae YC, et al. (2010) Effects of phosphoric acid treatment of titanium surfaces on surface properties, osteoblast response and removal of torque forces. Acta Biomater 6: 1661-70.

5. Li J, Liu X, Qiao Y, Zhu H, Li J, et al. (2013) Enhanced bioactivity and bacteriostasis effect of TiO, nanofilms with favorable biomimetic architectures on titanium surface. RSC Adv 3: 11214-25.

6. Frauchiger VM, Schlottig F, Gasser B, Textor M (2004) Anodic plasma-chemical treatment of CP surfaces for biomedical applications. Biomaterials 25: 593606.

7. Mändl S, Thorwarth G, Schreck M, Stritzker B, Rauschenbach B (2000) Raman study of titanium oxide layers produced with plasma immersion ion implantation. Surface and Coatings Technology 125: 84-8.

8. Minagar S, Berndt CC, Wang J, Ivanova E, Wen C (2012) A review of the application of anodization for the fabrication of nanotubes on metal implant surfaces. Acta Biomater 8: 2875-88.

9. Brammer KS, Frandsen CJ, Jin S (2012) $\mathrm{TiO}_{2}$ nanotubes for bone regeneration. Trends Biotechnol 30: 315-22.

10. Mor GK, Varghese OK, Paulose M, Shankar K, Grimes CA (2006) A review on highly ordered, vertically oriented TiO 2 nanotube arrays: Fabrication, material properties, and solar energy applications. Solar Energy Materials \& Solar Cells 90: 2011-75.

11. Omidvar H, Goodarzi S, Seif A, Azadmehr AR (2011) Influence of anodization parameters on the morphology of TiO nanotube arrays. Superlattices and Microstructures 50: 26-39. 
12. Gulati K, Ramakrishnan S, Sinn Aw M, Atkins GJ, Findlay DM, et al. (2012) Biocompatible polymer coating of titania nanotube arrays for improved drug elution and osteoblast adhesion. Acta Biomater 8: 449-56.

13. Macak JM, Tsuchiya H, Schmuki P (2005) High-aspect-ratio $\mathrm{TiO}_{2}$ nanotubes by anodization of titanium. Angew Chem Int Ed Engl 44: $2100-2$.

14. Tanaka SI, Fukushima Y, Nakamura I, Tanaki T, Jerkiewicz G (2013) Preparation and characterization of microporous layers on titanium by anodization in sulfuric acid with and without hydrogen charging. ACS Appl Mater Interfaces 5: 3340-7.

15. Kuromoto NK, Simão RA, Soares GA (2007) Titanium oxide films produced on commercially pure titanium by anodic oxidation with different voltages. Materials Characterization 58: 114-21.

16. Regonini D, Jaroenworaluck A, Stevensa R, Bowena CR (2010) Effect of heat treatment on the properties and structure of TiO 2 nanotubes: phase composition and chemical composition. Surf Interface Anal 42: 139-44.

17. Yang B, Uchidab M, Kimc HM, Zhang X, Kokubo T (2004) Preparation of bioactive titanium metal via anodic oxidation treatment. Biomaterials 25: 1003-10. 18. Oh S, Daraio C, Chen LH, Pisanic TR, Fiñones RR, et al. (2006) Significantly accelerated osteoblast cell growth on aligned TiO 2 nanotubes. J Biomed Mater Res A: 97-103.

19. Prato M, Alloisio M, Jadhav SA, Chincarini A, Svaldo-Lanero T, et al. (2009) Optical properties of disulfide-functionalized diacetylene self-assembled monolayers on gold: a spectroscopic ellipsometry study. J Phys Chem C 113: 20683-8.

20. Gomez Sanchez A, Schreiner W, Duffó G, Ceré S (2013) Surface modification of titanium by anodic oxidation in phosphoric acid at low potentials. Part 1. Structure, electronic properties and thickness of the anodic films. Surf Interface Anal 45: 1037-46.

21. Park YJ, Shin KH, Song HJ (2007) Effects of anodizing conditions on bond strength of anodically oxidized film to titanium substrate. Applied Surface Science 253: 6013-8.

22. Jaeggi C, Parlinska-Wojtan M, Kern P (2012) Correlation of electrolyte-derived inclusions to crystallization in the early stage of anodic oxide film growth on titanium. Thin Solid Films 520: 1804-8.

23. Sedona F, Rizzi GA, Agnoli S, Llabre's i Xamena FX, Papageorgiou A, et al. (2005) Ultrathin TiOx Films on Pt(111): A LEED, XPS, and STM investigation. J Phys Chem B 109: 24411-26.

24. Matsumoto T, Batzill M, Hsieh S, Koel BE (2004) Fundamental studies of titanium oxide-Pt(100) interfaces I. Stable high temperature structures formed by annealing TiOx films on Pt(100). Surface Science 572: 127-45.

25. Bardi U (1990) On the composition and structure of thin layers of titanium oxide on platinum surfaces. Catalysis Letters 5: 81-8.

26. Toccafondi C, Uttiya S, Cavalleri O, Gemme G, Barborini E, et al. (2014) Optical properties of nanogranular and highly porous TiO thin films. J Phys D: Appl. Phys., submitted

27. Zhao D, Chen C, Wang Y, Ji H, Ma W, et al. (2008) Surface Modification of TiO 2 by Phosphate: Effect on Photocatalytic Activity and Mechanism Implication. J Phys Chem C 112: 5993-6001.

28. Marino CEB, Nascente PAP, Biaggio SR, Rocha-Filho RC, Bocchi N (2004) XPS characterization of anodic titanium oxide films grown in phosphate buffer solutions. Thin Solid Films 468: 109-12.

29. Lottici PP, Bersani D, Braghini M, Montenero A (1993) Raman scattering characterization of gel- derived titania glass. J Mater Sci 28 : $177-83$.

30. Balachandran U, Eror NG (1982) Raman spectra of titanium dioxide. Journal of Solid State Chemistry 42: 276-82.

31. Krasicka-Cydzik E, (2012) Anodic Layer Formation on Titanium and Its Alloys for Biomedical Applications, in Titanium Alloys - Towards Achieving Enhanced Properties for Diversified Applications, Nurul Amin AKM Ed., Intech Publisher.

32. Charnley M, Textor M, Acikgoz C (2011) Designed polymer structures with antifouling-antimicrobial properties. Reactive Functional Polymers 71: 329-34.

33. Thorat S, Diaspro A, Scarpellini A, Povia M, Salerno M (2013) Comparative study of loading of anodic porous alumina with silver nanoparticles using different methods. Materials 6: 206-16.

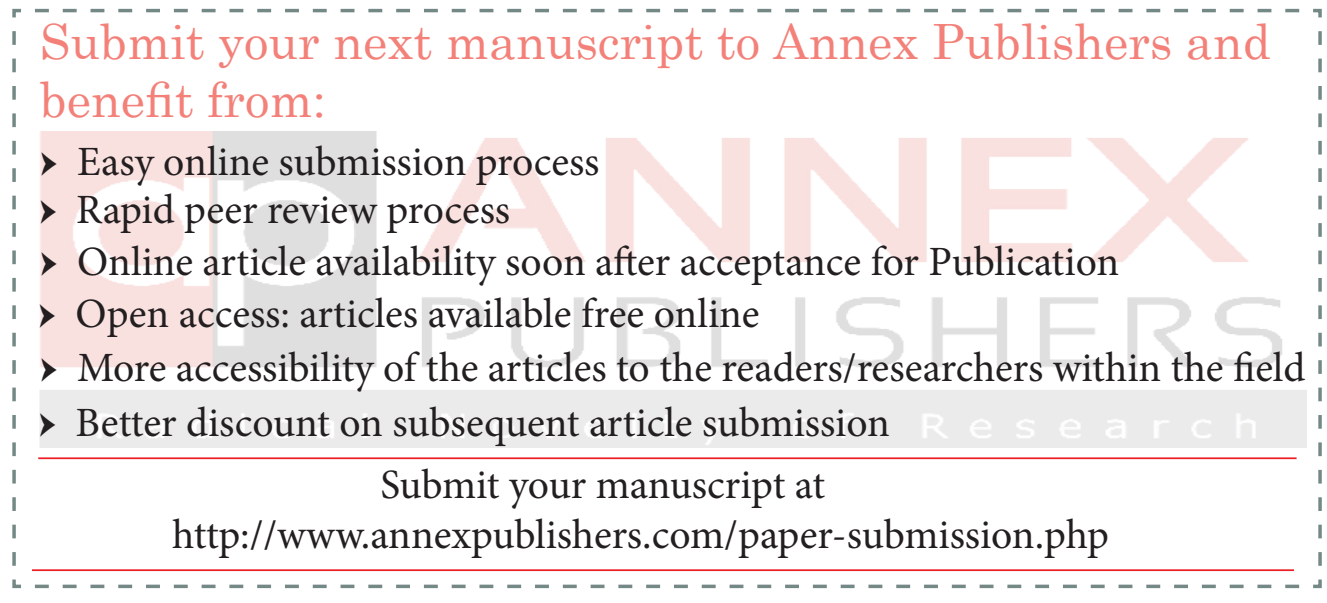

